\title{
Human African trypanosomiasis (sleeping sickness) in a Nigerian male adolescent and the treatment challenges: A case report
}

\author{
Chizoma I. Eneh ${ }^{1}$, Samuel N. Uwaezuoke ${ }^{* 2}$, Henrietta U. Okafor ${ }^{2}$, Benedict O. Edelu ${ }^{2}$, F.N. Ogbuka ${ }^{1}$ \\ ${ }^{1}$ Enugu State University Teaching Hospital (Park-lane), Enugu, Nigeria \\ ${ }^{2}$ University of Nigeria Teaching Hospital (UNTH), Enugu, Nigeria
}

\author{
Received: December 13, $2015 \quad$ Accepted: January 13, $2016 \quad$ Online Published: January 16, 2016 \\ DOI: $10.5430 /$ jer.v2n2p31 \\ URL: http://dx.doi.org/10.5430/jer.v2n2p31
}

\begin{abstract}
Human African Trypanosomiasis (HAT) or "Sleeping Sickness" is a potentially fatal but curable, neglected tropical disease seen in sub-Saharan Africa. HAT was nearly eliminated in the 1960s, but has re-emerged in epidemic proportions over the past two decades. We report a case of HAT in order to highlight a possible re-emergence of this rare tropical disease, as well as its treatment challenges. A.C is a 14-year-old male patient who presented with clinical features suggestive of the chronic form of the disease. Despite the prescription of IV Eflornithine, the drug could not be procured. Unfortunately, the patient was lost to follow-up. This treatment gap underscores the need for emphasis on preventive measures such as eradication of tsetse flies, and reduction of the spread of parasites especially in resource-limited settings of sub-Saharan Africa.
\end{abstract}

Key Words: Human African Trypanosomiasis, Treatment challenges, Sub-Saharan Africa

\section{INTRODUCTION}

Human African Trypanosomiasis (HAT) or "Sleeping Sickness" is a potentially fatal but curable, neglected tropical disease which is caused by the protozoan parasite: Trypanosoma brucei gambiense (associated with the chronic illness) and Trypanosoma brucei rhodesiense (associated with the acute illness). ${ }^{[1]}$ The parasite is transmitted to humans through the bite of infected tsetse flies which breed in warm and humid areas. Transmission may also occur through the transfusion of blood and blood products or from mother-to-child through the placenta. Tsetse flies are found only in Sub-Saharan Africa, which puts about 60 million people at risk. ${ }^{[2]}$

HAT was nearly eliminated in 1960 s by colonial control pro- grammes, ${ }^{[3]}$ but has re-emerged in epidemic proportions due to wars, population movements and the collapse of healthcare systems over the past two decades. ${ }^{[4]}$ According to the World Health Organisation (WHO) report in $1998,{ }^{[3]} 300,000$ to 500,000 people were affected per year with 60,000 dying per year. The incidence has however declined progressively to $<10,000$ new cases per year since 2010 ${ }^{[5]}$ Between 2008 to 2009 Nigeria was among the 10-12 African countries reporting fewer than 100 new cases per year, while Democratic Republic of Congo and Central African Republic reported $>1,000$ new cases per year. ${ }^{[5]}$ Disease prevalence differs from one country to another and in different parts of a single country. Currently, the prevalence is 30,000 , although WHO estimates that the true number of cases was at least 10 times Nigeria.

*Correspondence: Samuel N. Uwaezuoke; Email: snuwaezuoke@yahoo.com; Address: Department of Pediatrics, UNTH Ituku-Ozalla, Enugu, 
more than that reported. ${ }^{[3-5]}$ Thus, the re-emergence of HAT presents a major public health problem.

HAT occurs mainly in large areas of savannah, in cattle settlements and in areas with wild game reserves, where tsetse flies are prevalent and animals serve as reservoir of the parasite. HAT may also be found in forests, lakes and water courses. ${ }^{[6]}$ The most exposed population comprises rural dwellers who are involved in agriculture, animal husbandry and hunting.

Some authors recently analyzed data from the epidemiological section of the Nigerian Institute for Trypanosomiasis Research (NITR) and reported that the mortality rate for $1970-1979$ was $0.003 \%$ out of approximately 28,000 infected persons. ${ }^{[7]}$ The peak rate occurred in 1974 with $18.1 \%$ deaths followed by 1972 with $17.7 \%$ deaths. ${ }^{[7]}$ With the decline of infection rates over the years, the authors suggest that the overwhelming success achieved in the control of the disease in the past appeared to have affected the way governments perceived the disease, as what followed was dwindling funding over several decades, resulting in the breakdown of surveillance and vector control activities. ${ }^{[7]}$ Furthermore, the ecological conditions that could result in epidemic outbreak of HAT are still present till date. Despite its endemic nature, only few case reports on HAT from the country exist in the literature. One case report was about HAT in a pregnant woman who was successfully treated with Melarsoprol. ${ }^{[8]}$ Most reports were about imported HAT among Caucasians who had visited African countries in the past. ${ }^{[9-12]}$ We report a case of HAT in a Nigerian patient in order to highlight a possible re-emergence of the disease, as well as its treatment challenges.

\section{CASE REPORT}

A.C was a 14-year-old male, born and bred in the urban setting of Enugu metropolis, capital of former eastern region of Nigeria. He came from Obeagu, a rural setting in Nkanu local government area (a few kilometers' drive from Enugu metropolis), where he used to spend an average of two weeks per year (during the Christmas holidays) in the last 5 years. He was the first of five children, four boys and one girl. He presented at the Children Outpatient Clinic of the University of Nigeria Teaching Hospital (UNTH) Enugu with history of excessive daytime sleepiness (EDS), first at rest and then while walking, which had lasted five years, and aggressiveness and worsening of academic performance that lasted for four years.

There were no recollections of early symptoms, such as skin lesions or non-specific symptoms, such as febrile episodes, headache, sweating, and night insomnia. However, patient had lost some weight during the course of the illness. There was no history of drug use, epilepsy or head trauma prior to the onset of an illness.

Physical examination revealed a patient, who was frequently sleeping off during a review, but was easily arousable. He was pale, and had a significant peripheral lymphadenopathy. Gait was normal on ambulation. Patient displayed no aggressiveness or mood changes. There were no other abnormal neurologic findings. An initial diagnosis of HAT ("sleeping sickness") was made.

Microscopy of dried Giemsa-stained thick blood film showed intermediate and stumpy forms of Trypanosoma brucei species (see Figure 1). There was evidence of hemolysis with anemia, neutropenia and lymphocytosis on complete blood count and blood smear. Electro-encelogram (EEG) showed no epileptiform discharges, with non-specific bipolar montage of mostly right hemisphere. Cerebro-spinal fluid biochemistry was non-specific, while Gram and Leishmann stains of CSF were normal.

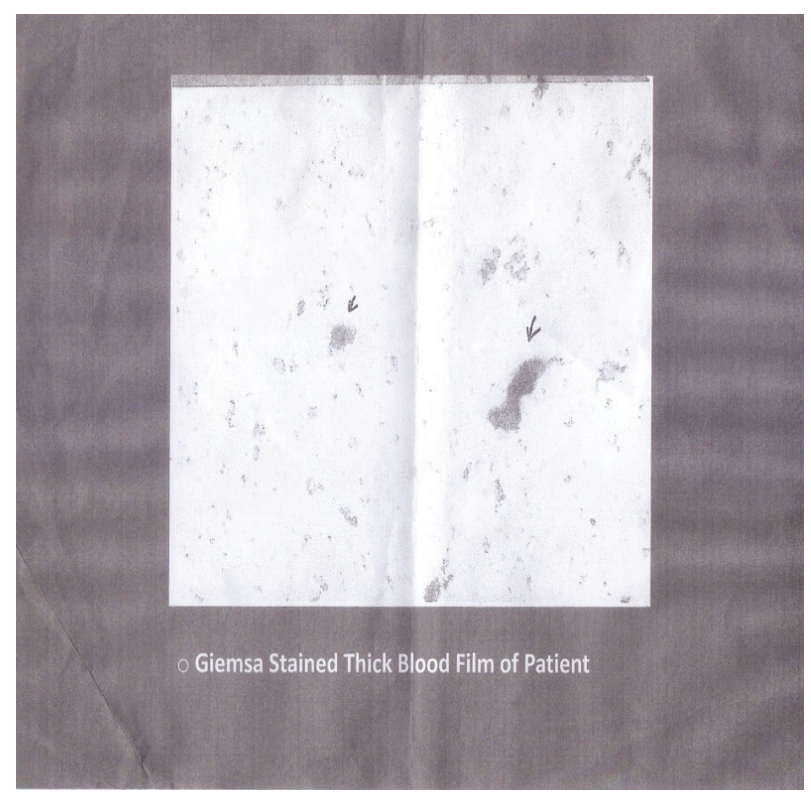

Figure 1. Microscopy of dried Giemsa-stained thick blood film, showing intermediate and stumpy forms of Trypanosoma brucei species (arrowed)

A definitive diagnosis of HAT was made and the patient was prescribed IV Eflornithine $50 \mathrm{mg} / \mathrm{kg}$ every 6 hours for 14 days. A year after the patient's first contact with the health facility, the drugs could not be procured. The State and Federal Ministries of Health were notified. Although frantic efforts were made to procure the drugs, the patient was unfortunately lost to follow-up. 


\section{DISCUSSION}

West African HAT caused by Trypanosoma brucei gambiense is usually contracted close to settlements, requiring only a small vector population. Thus, it is particularly difficult to be eradicated. Because of a low-level of parasitemia exhibited during HAT, transmission requires close and repeated human-and-fly contacts. Our patient might have been exposed to tsetse flies during any of his yearly visits to his rural residence, a farm-settlement with a small river and some cattle-rearing activities. In a study of the prevalence and risk factors of HAT in an endemic focus in Nigeria, some investigators reported human activities such as farming and visits to the river as major risk factors. ${ }^{[13]}$ Their findings confirm our patient's exposure to epidemiologic risk factors for HAT.

HAT has three stages: hard painful nodules/chancre with surrounding erythema and swelling 2-3 days after tsetse-fly bite. This subsides spontaneously in 2 weeks, leaving no permanent scar. Hemo-lymphatic stage is the stage of invasion of the lymphatic system and the blood stream 2-3 weeks later. This stage is characterized by irregular episodes of fever, fatigue, headache, increased sweating, generalized lymphadenopathy, weight loss and night insomnia. These episodes are separated by symptom-free period of 1-7 days The patient had no clear recollection of such features. At the meningo-encephalitic stage, the patient was irritable, had night insomnia, anxiety, mood and personality changes with progressive drowsiness, which ended up in uncontrollable urge to sleep, first during the day and terminally day and night. HAT was named after this chronic diffuse meningoencephalitic stage without localizing signs. The patient exhibited periods of intense sadness, anxiety and aggression towards his siblings and peers, in addition to the sleeping disorder. In the Trypanosoma brucei gambiense infestation (West African trypanosomiasis/chronic form), the meningoencephalitic stage appears within two years after the onset of acute symptoms. ${ }^{[14]}$ This differs from the Trypanosoma brucei rhodesiense infestation (East African trypanosomiasis/acute form), in which CNS invasion occurs within 3-6 weeks with death, usually from secondary infection or cardiac failure occurring in 6-9 months. Our patient clearly had the chronic form of HAT. In our patient, the confirmatory diagnosis was established by the finding of the intermediate and stumpy forms of Trypanosoma brucei species on microscopic examination of dried Giemsa-stained thick blood film. The two species are morphologically indistinguishable, but Trypanosoma brucei gambiense is responsible for $95 \%$ of reported cases. The diagnosis of HAT requires the detection of the parasite by blood film mainly using concentration techniques. ${ }^{[15]}$ The findings in the patient's complete blood count

Published by Sciedu Press were in accordance with HAT, however the CSF findings were not specific, as was noted in a previous report, ${ }^{[16]}$ while serological tests for parasite and its antibody detection could not be performed. However, it has been noted that serological tests such as card agglutination test for trypanosomiasis are rather complimentary to the classical parasitological procedures in the diagnosis of West African Trypanosomiasis and therefore serve rather as screening tools. ${ }^{[17]}$

Eflornithine has been effective in the late stage of West African trypanosomiasis (suspected/confirmed CNS invasion). ${ }^{[18]}$ It is more effective against Trypanosoma brucei gambiense, and variably effective against Trypanosoma brucei rhodesiense. Melarsoprol is a more toxic alternative used when there is also CNS invasion. ${ }^{[19]}$ Toxic effects include encephalopathy, psychotic reactions, brain damage, and exfoliative dermatitis. ${ }^{[19]}$ Nevertheless, Melarsoprol has been successfully used in a pregnant woman with HAT without toxic effect to her and her baby after 2 years of follow-up. ${ }^{[8]}$ Other current standard treatments include IV/IM Pentamidine, which is first line drug for hemo-lymphatic stage of Trypanosoma brucei gambiense infection, while IV Suramin is suitable for Trypanosoma brucei rhodesiense infection.

More recently, Eflornithine and Nifurtimox are being tried as a combination therapy for a safer, easier to administer and more effective regimen. ${ }^{[20,21]}$ Patient is treated with $7.5 \mathrm{mg} / \mathrm{kg}$ Nifurtimox for 3-10 days plus Eflornithine IV 50 $\mathrm{mg} / \mathrm{kg}$ every 6 hours for 14 days. There are different ongoing researches for newer, safer and more effective drugs, but antigenic variability (> 800 genes) of Trypanosoma brucei makes it evade immune system detection.

We had a challenge in treating our patient despite the prescription of Eflornithine; the drug could not be procured from the hospital and from the state authorities. Due to the treatment delay, patient was eventually lost to follow-up. This experience is in contrast to the prompt treatment of the pregnant woman with HAT in another part of the country. ${ }^{[8]}$ This patient was managed at a mission hospital (Eku Baptist Hospital) in Delta state, the only state where transmission was then occurring in Nigeria, specifically the Abraka focus. ${ }^{[8]}$ Expectedly, drugs from government and donor agencies were possibly not in short supply in this endemic region.

Elsewhere in European countries not endemic for HAT, diagnosis of imported cases of infection with Trypanosoma brucei gambiense is challenging because of variations in clinical features and low sensitivities of diagnostic tests. ${ }^{[9]}$ Two cases of imported Trypanosoma brucei gambiense were reported in a 44 year-old male and 54 year-old female, who had lived and worked as expatriates in Gabon and Central African Republic, respectively. ${ }^{[9]}$ Similar to our experience, 
the challenges in the management of these patients were delayed procurement of Eflornithine from the WHO due to logistic issues, as well as initial misdiagnosis because of the nature of clinical presentation. ${ }^{[9]}$ Other case reports of imported HAT have been documented in Caucasian subjects, who had visited Central Africa, ${ }^{[10]}$ and West Africa. ${ }^{[11,12]}$ In one of the reports, ${ }^{[12]}$ a 12-year-old European girl, who lived in the West African country of Senegal for 9 months presented with West African trypanosomiasis. Diagnosis was made because of the occurrence of uveo-meningitis, following progressive alteration of her general condition. In contrast to our experience with the index patient, this girl had a favorable outcome after two courses of Eflornithine (diflouromethylornithine, DFMO). This observation highlights the disparity in the availability of anti-HAT drugs between the developed and developing countries.

\section{Conclusions}

The WHO is collaborating with a number of pharmaceutical companies to produce and donate large quantities of Eflornithine, especially to resource-poor developing countries. Researchers/doctors with Medicines Sans Frontiers have cur- rently taken up the challenge of supplying drugs for the treatment of HAT. Authorities in sub-Saharan African countries should work in synergy with such non-governmental organizations (NGOs) to ensure availability of and accessibility to these drugs.

Furthermore, there is on-going research to produce drugs against the organism's flagella protein with which it needs to survive in the human blood stream.

The treatment gap noted in this report underscores the need for emphasis on preventive measures, such as eradication of tsetse flies, and reduction of the spread of the parasites, especially in the resource-limited settings of sub-Saharan Africa. This can be achieved by the use of sterile insect technique. There are also medical/veterinary strategies, which help to reduce the spread of parasites such as monitoring, prophylaxis, and treatment of infected people and animals.

\section{Limitation}

The serological tests for the parasite and its antibody detection could not be done due to their non-availability in our laboratory facility.

\section{REFERENCES}

[1] Dumas M, Bouteille B, Buguet A (Eds). Progress in Human African Trypanosomiasis, Sleeping Sickness. Paris: Springer-Verlag. 1999 http: //dx .doi .org/10.1007/978-2-8178-0857-4

[2] Epidemiology and Control of African Trypanosomiasis: Report of a WHO expert committee. World Health Organization (1986) Geneva: Switzerland, Technical Report Series No. 739: 126.

[3] World Health Organisation: Control and Surveillance of African Trypanosomiasis. Geneva: WHO. 1998.

[4] WHO report on global surveillance of epidemic prone infectious diseases-African Trypanosomiasis. 2001.

[5] Simarro PP, Diarra A, Ruiz Postigo JA, et al. The Human African Trypanosomiasis control and surveillance programme of the World Health Organisation 2000-2009: The way forward. PLOS Negl Trop Dis. 2011; 5(2): e1007. PMid:21364972 http: //dx.doi .org/10. 1371/journal.pntd. 0001007

[6] Fèvre EM, Coleman PG, Odiit M, et al. The origins of a new Trypanosoma brucei rhodesiense sleeping sickness outbreak in eastern Uganda. Lancet. 2001; 358(9282): 625-628. http://dx.doi .org /10.1016/S0140-6736(01) 05778-6

[7] Anere JI, Fajinmi AO, Lawani FAG. An analysis of Human African Trypanosomiasis (HAT) in Nigeria. Science World Journal. 2006; 1(1).

[8] Ugoji UC, Bock-Oruma AA, Umukoro D, et al. Human African Trypanosomiasis successfully treated with Melarsoprol in pregnancy in a Niger Delta rural hospital. Case Reports in Clinical Medicine. 2014; 3: 353-356. http://dx.doi.org/10.4236/crcm. 2014.36078

[9] Bisoffi Z, Beltrame A, Monteiro G, et al. African trypanosomiasis gambiense, Italy. Emerg Infect Dis. 2005; 11(11): 1745 1747. PMid:16318728 http://dx.doi.org/10.3201/eid1111 .050649
[10] Iborra C, Danis M, Bricaire F, et al. A traveller returning from Central Africa with fever and a skin lesion. Clin Infect Dis. 1999; 28(3): 679680. PMid:10194096 http://dx . doi .org/10.1086/517213

[11] Grau Junyent JM, Rozman M, Corachan M, et al. An unusual course of west African trypanosomiasis in a Caucasian man. Trans R Soc Trop Med Hyg. 1987; 81(6): 931-932. http://dx.doi.org/10. 1016/0035-9203(87) 90356-7

[12] Buissonniere RF, de Boisseieu D, Tell G, et al. Uveo-meningitis revealing a West African trypanosomiasis in a 12 year-old girl. Arch Fr Pediatr. 1989; 46(7): 517-519. PMid:2512891

[13] Onyebiguwa PGN, Clement I, Igho BI, et al. Human African trypanosomiasis in endemic focus of Abraka, Nigeria. Asian Pacific $\mathbf{J}$ Trop Med. 2010; 3(6): 448-450. http://dx . doi .org/10.1016/S $1995-7645$ (10)60107-1

[14] Greenwood BM, Whittle HC. The pathogenesis of sleeping sickness. Trans R Soc Trop Med Hyg. 1980; 74(6): 716-725. http: //dx.doi.org/10.1016/0035-9203(80)90184-4

[15] Bailey JW, Smith DH. The use of the acridine orange QBC technique in the diagnosis of African Trypanosomiasis. Trans R Soc Trop Med Hyg. 1992; 86: 630. http://dx.doi.org/10.1016/0035-920 3(92) 90160-E

[16] Jamonneau V, Solano P, Garcia A, et al. Stage determination and therapeutic decision in human African trypanosomiasis: value of polymerase chain reaction and immunoglobulin $\mathrm{M}$ quantification on the cerebrospinal fluid of sleeping sickness patients in Côte d'Ivoire. Trop Med. Int Health. 2003; 8(7): 589-594. PMid:12828540 http://dx.doi.org/10.1046/j.1365-3156.2003.01079.x

[17] Truc P, Bailey JW, Doua F, et al. A comparison of parasitological methods for the diagnosis of gambian trypanosomiasis in an area of low endemicity in Côte d'Ivoire. Trans R Soc Trop Med Hyg. 1994; 
88(4): 419-421. http://dx.doi.org/10.1016/0035-9203(94 ) $90410-3$

[18] Sjoerdsma A, Schechter PJ. Eflornithine for African sleeping sickness. Lancet. 1999; 354(9174): 254. http://dx. doi .org/10.10 16/S0140-6736(05) 66324-6

[19] Dumas M, Bouteille B. Treatment of human African trypanosomiasis. Bull World Health Organ. 2000; 78(12): 1474. PMid:11196500

[20] Pepin J, Milord F, Mpia B, et al. An open clinical trial of nifurtimox for arseno-resistant Trypanosoma brucei gambiense sleeping sickness in central Zaire. Trans Roy Soc Trop Med \& Hyg. 1989; 83(4): 514 517. http://dx.doi .org/10.1016/0035-9203(89) 90270-8

[21] Priotto G, Kasparian S, Mutombo W, et al. Nifurtimox-eflornithine combination therapy for second-stage African Trypanosoma brucei gambiense trypanosomiasis: a multicenter, randomized, phase III, non-inferiority trial. Lancet. 2009; 374(9683): 56-64. http: //dx.doi.org/10.1016/S0140-6736(09)61117-X 